\title{
Propiedades psicométricas del Cuestionario de Admiración y Rivalidad Narcisista (NARQ) en estudiantes universitarios de Venezuela
}

\section{Psychometric properties of the Narcissistic Admiration and Rivalry Questionnaire (NARQ) in college students from Venezuela}

\section{Propriedades psicométricas do Questionário de Admiração e Rivalidade de Narcisistas (NARQ) em estudantes universitários da Venezuela}

\author{
Daniel Chaustre Jota, ORCID 0000-0002-1535-4202 \\ Centro de Investigación y Evaluación Institucional (CIEI), Universidad Católica Andrés \\ Bello (UCAB), Venezuela
}

Resumen: La investigación tuvo como objetivo analizar las propiedades psicométricas y la adecuación del Cuestionario de Admiración y Rivalidad Narcisista (NARQ) en una muestra no probabilística de estudiantes universitarios $(\mathrm{N}=159 ; 74 \%$ mujeres $)$ de Caracas (Venezuela), pertenecientes a una universidad privada. A través del método bola de nieve se recabó la información por medio de la administración de un cuestionario en línea. Mediante el análisis factorial exploratorio se comprobó la solución factorial propuesta, también se hallaron relaciones significativas entre admiración y rivalidad con autoestima, extraversión, apertura y afabilidad, tal como se reporta en la literatura. Se concluye que el NARQ presenta evidencias de validez y confiabilidad, y por tanto se recomienda su uso en la población venezolana.

Palabras clave: personalidad narcisista; narcisismo; admiración; rivalidad; adaptación.

Abstract: The research had as primary objective to analyze the psychometric properties and the adequacy of the Narcissistic Admiration and Rivalry Questionnaire (NARQ) in a nonprobability sample of college students $(\mathrm{N}=159 ; 74 \%$ women $)$ from a private university in Caracas (Venezuela). Through a snowball procedure, the data were collected via application of an online questionnaire. Using an exploratory factor analysis, the proposed factorial solution was obtained; also, significant correlations were found between admiration and rivalry with self-esteem, extraversion, openness and agreeableness, just as stated in the literature. The NARQ presents evidence of validity and reliability, and therefore its use could be recommended in the Venezuelan population.

Keywords: narcissistic personality; narcissism; admiration; rivalry; adaptation.

$(c)$ EY

Esta obra está bajo una licencia de Creative Commons Reconocimiento 4.0 Internacional 
Resumo: O objetivo da pesquisa foi analisar as propriedades psicométricas e a adequação do Narcissistic Admiration and Rivalry Questionnaire (NARQ) em uma amostra não probabilística de estudantes universitários ( $\mathrm{N}=159 ; 74 \%$ mulheres) de Caracas (Venezuela), pertencentes a uma universidade privada. Utilizando o método bola de neve, as informações foram coletadas por meio da aplicação de questionário online. Mediante análise fatorial exploratória, confirmou-se a solução fatorial proposta, também foram encontradas relações significativas entre admiração e rivalidade com autoestima, extroversão, franqueza e simpatia, assim como se descreve na literatura. Conclui-se que o NARQ apresenta evidências de validade e confiabilidade, portanto, seu uso é recomendado na população venezuelana.

Palavras-chave: personalidade narcisista; narcisismo; admiração; rivalidade; adaptação.

Recibido: 04/03/2021

Aceptado: 31/08/2021

Cómo citar:

Chaustre Jota, D. (2021). Propiedades psicométricas del Cuestionario de Admiración y Rivalidad Narcisista (NARQ) en estudiantes universitarios de Venezuela. Ciencias Psicológicas, 15(2), e-2484. doi: https://doi.org/10.22235/cp.v15i2.2484

Correspondencia: Daniel Chaustre Jota, Centro de Investigación y Evaluación Institucional (CIEI), Universidad Católica Andrés Bello (UCAB), Venezuela.E-mail: dchaustr@ucab.edu.ve

El narcisismo es un constructo de personalidad con una larga historia dentro de la psicología. A partir de la década de 1980 se produjeron una serie de eventos relacionados con la investigación alrededor de este constructo: se presentó un incremento en la prevalencia de síntomas narcisistas reportados por psicólogos y analistas (Tyler, 2007), asimismo se incluyó a la personalidad narcisista como un trastorno de personalidad dentro del manual diagnóstico y estadístico de las enfermedades mentales (DSM, por sus siglas en inglés de la American Psychiatric Association, 1980). A partir de esa década surgen importantes innovaciones en la medición del narcisismo. En adición a la entrevista clínica y a la observación, comenzaron a utilizarse cuestionarios estandarizados de autorreporte, los cuales han sido estudiados y validados tanto en poblaciones clínicas como en poblaciones no clínicas (Emmons, 1987).

La sistematización de los resultados obtenidos, producto de esta nueva dirección en la investigación del narcisismo, trajo consigo nuevos avances, especialmente por parte de la psicología social y de la personalidad, cuyos aportes han permitido comprobar o rechazar ciertas asunciones derivadas de las investigaciones clínicas sostenidas en el pasado (Levy, Ellison \& Reynoso, 2011). Surgió entonces una nueva forma de comprender a un constructo heterogéneo, cuya caracterización fluctuaba marcadamente entre la psiquiatría, la psicología clínica y la psicología social y de la personalidad (Pincus \& Roche, 2011), discrepancias que implicaban consecuencias sociales diferentes para estos rasgos. Conceptualizar al narcicismo como un constructo bidimensional permitió comprender parte de las razones por las cuales 
se encontraba tanta heterogeneidad entre las investigaciones, ya que ambos componentes comparten características nucleares, pero sus rasgos asociados difieren considerablemente (Wink, 1991).

En la década de 2010, otras posturas teóricas han emergido para dar explicaciones más completas, incluyendo además nuevas formas de medir el narcisismo. Si bien existen algunos cuestionarios ampliamente utilizados desde su aparición, como es el caso del Inventario de Personalidad Narcisista (NPI, por sus siglas en inglés; Raskin \& Hall, 1979; Raskin \& Terry, 1988) y de la Escala de Narcisismo Hipersensible (HSNS, por sus siglas en inglés; Hendin \& Cheek, 1997), hace algunos años aparecieron novedosas formas de medir el constructo, en sintonía con los avances de la teoría subyacente (Rogoza, ZemojtelPiotrowska \& Campbell, 2019).

Una de estas recientes posturas teóricas se denomina Concepto de Admiración y Rivalidad Narcisista (NARC, por sus siglas en inglés; Back et al., 2013). Esta tiene como supuesto que las personas categorizadas como narcisistas persiguen una meta particular: mantener una visión grandiosa de sí mismos. Las maneras en que se puede alcanzar este objetivo son variadas, la propuesta del modelo NARC establece que existen dos estrategias generales, denominadas rivalidad y admiración narcisista, respectivamente.

Inicialmente, la estrategia predilecta en las personas narcisistas es la admiración. Esta se caracteriza por la tendencia a promover una imagen positiva de sí mismas, a través de la admiración social que se obtiene al realzarse frente a otros (Back, 2018). Mostrarse habladores y señalar constantemente sus cualidades positivas y sus logros son algunas de las características que pueden encontrarse en esta estrategia. Los resultados asociados a esta aproximación suelen ser positivos a corto plazo; sin embargo, a largo plazo el reconocimiento y el agrado por parte de otras personas suele decaer de manera considerable (Wurst et al., 2017).

La segunda estrategia empleada es la rivalidad narcisista. Esta se caracteriza por la tendencia a protegerse de una visión negativa de sí mismo, mediante la devaluación de otros, mostrando además comportamientos egoístas, socialmente insensibles, arrogantes y hostiles, asimismo exhiben falta de confianza, de afecto interpersonal y de perdón hacia otros (Wurst et al., 2017). En estos casos, el individuo opta por denigrar y desvalorar a aquellas personas con las que compite por estatus y reconocimiento; menospreciando públicamente las cualidades de otras personas, realza las suyas. Esta estrategia, a diferencia de la admiración, suele conllevar resultados negativos tanto a corto como a largo plazo (Wurst et al., 2017).

La predilección o disposición de un individuo a utilizar una u otra estrategia es también un punto de discusión en la actualidad. Tal parece que ciertas características de personalidad inclinan la balanza hacia un lado o hacia el otro, así como las posibles claves sociales del entorno, y la retroalimentación que otras personas brinden al empleo de una estrategia particular (Back, 2018).

En cuanto a la relevancia de los rasgos de personalidad, se han encontrado las siguientes relaciones (Back et al., 2013; Leckelt et al., 2017): la extraversión suele estar asociada con ambas estrategias, pero en direcciones opuestas. Individuos más extrovertidos son más proclives a emplear la estrategia de admiración, mientras que la rivalidad suele asociarse negativamente con este rasgo. Situación similar se produce con el neuroticismo, sin embargo, en este caso es la admiración la cual se asocia negativamente con este rasgo de personalidad, mientras que la relación con la rivalidad narcisista resulta positiva. Otra 
característica personal que se ha asociado de manera diferenciada con estas estrategias es la autoestima. Específicamente, se ha encontrado que la admiración tiene una correlación positiva con la autoestima, y, por su parte, la rivalidad presenta una correlación negativa (Geukes et al., 2017).

Asimismo, otro factor determinante en el empleo de las estrategias se refiere a las consecuencias sociales que estas tienen sobre el individuo narcisista. Consecuencias sociales positivas refuerzan el uso de la estrategia de admiración; en el caso de no obtener el reconocimiento esperado, la persona suele optar en cambio por denigrar a otros a través de la rivalidad (Back, 2018). Esta distinción en cuanto a la manera en que una persona narcisista se maneja en sus entornos sociales resulta de gran importancia. Aunque los rasgos de fondo permanecen estables, la manera en que un individuo se comporta para alcanzar un mismo objetivo: el estatus social, puede cambiar de manera considerable, produciendo distintos resultados que pueden afectar, por una parte, su permanencia dentro de un grupo (Benson, Jeschke, Jordan, Bruner \& Arnocky, 2018), así como las relaciones con otras personas (Wurst et al., 2017). El cuestionario desarrollado en consonancia con el modelo NARC (Back et al., 2013) permite evaluar ambos aspectos al mismo tiempo.

El Cuestionario de Admiración y Rivalidad Narcisista (Back et al., 2013) es una prueba desarrollada para evaluar los elementos asociados a las estrategias de rivalidad y admiración. En ambos casos evalúa además los elementos cognitivos, afectivos y conductuales asociados a estas estrategias. Es por esto que resulta un instrumento de gran utilidad en la evaluación de la personalidad. Siendo además un instrumento corto que ha mostrado resultados psicométricamente adecuados en diferentes contextos y poblaciones (Doroszuk et al., 2019).

En este sentido, el NARQ es un instrumento que permite estudiar dos dimensiones del narcisismo, considerando al mismo tiempo las motivaciones subyacentes, así como los resultados conductuales y las dinámicas interpersonales de ambas (Leckelt et al., 2017). Esta es una de las principales ventajas del cuestionario, especialmente en comparación con otros instrumentos más empleados, como el NPI, que ha demostrado una escasa cobertura de los aspectos mal adaptativos del narcisismo (Leckelt et al., 2017).

Recientemente han tenido lugar adaptaciones del cuestionario a distintos idiomas, demostrando adecuadas propiedades psicométricas. Pueden encontrarse versiones en: a) polaco, donde ha presentado indicadores adecuados de confiabilidad, de validez externa al correlacionar con otros instrumentos que miden narcisismo, y de validez factorial, comprobada a partir de un análisis factorial confirmatorio (Rogoza, Rogoza, \& Wyszynska, 2016); b) italiano, donde se pudo comprobar, mediante un análisis confirmatorio, la estructura de dos factores, así como la confiabilidad y la validez externa del instrumento, tras la correlación diferencial con autoestima y los cinco grandes (Vecchione et al., 2018); y, finalmente, c) español (Doroszuk et al., 2019).

Con respecto a la validación realizada por Doroszuk et al. (2019) para la versión en español, esta fue llevado a cabo en Colombia, Chile y España, donde se hallaron coeficientes de confiablidad alfa de Cronbach $(\alpha)$ adecuados, tanto para admiración (mediana $\alpha=.81$ ) como para rivalidad (mediana $\alpha=.81$ ), así como indicadores de validez externa a través de patrones de asociación diferenciados con variables como envidia y autoestima. Puede apreciarse que el instrumento, en sus adaptaciones para ajustarse a un idioma particular, ha 
demostrado propiedades adecuadas; sin embargo, aún es necesario realizar una investigación para evaluar las propiedades del instrumento específicamente en poblaciones venezolanas.

Es por esto que el objetivo de la investigación está dirigido a realizar una primera aproximación a la validación el instrumento, al estudiar las propiedades psicométricas el NARQ en una muestra venezolana de estudiantes universitarios, comprobando de esta manera que presente los adecuados índices de confiabilidad y validez para ser empleada en investigaciones futuras. Asimismo, se propone describir las diferencias en poblaciones venezolanas en comparación con otros países hispanohablantes.

\section{Método}

\section{Participantes}

La muestra estuvo constituida por 159 estudiantes de una universidad privada en Caracas (Venezuela). De estos, 118 eran mujeres (74\%), el resto hombres. La edad promedio de los participantes fue de 20.92; con una desviación típica de 2.24 años $(C V=10.7 \%)$. El criterio de inclusión considerado para formar parte de la muestra fue estar matriculado en alguna de las carreras de pregrado ofrecidas por la universidad, durante el período académico vigente en el momento de la recolección de los datos. La selección de los sujetos se realizó mediante un proceso no probabilístico, por el método de bola de nieve (Peña, 2017).

El tamaño muestral propuesto inicialmente fue de 180 personas, siguiendo las sugerencias de Hair, Black, Babin y Anderson (2009) de contar con 10 casos por variable para realizar el análisis factorial, considerando que el NARQ cuenta con un total de 18 ítems. Sin embargo, esta cifra no pudo alcanzarse durante la recolección; a pesar de esto, se cumplió el criterio mínimo de Hair et al. (2009) de contar con una muestra mayor a 100 casos, y de tener al menos 5 sujetos por variable. La participación fue completamente voluntaria y los sujetos no recibieron ningún tipo de compensación por su participación en la investigación.

\section{Instrumentos}

Narcissistic Admiration and Rivalry Questionnaire (NARQ)

Es un cuestionario de 18 ítems, con una escala de respuesta tipo Likert, que va desde 1 (completamente en desacuerdo) hasta 6 (completamente de acuerdo). La escala está dividida en dos estrategias generales, las cuales se dividen a su vez en tres subcomponentes, conformados por tres ítems cada uno. Las estrategias generales son admiración y rivalidad. La primera de ellas está conformada por: a) grandiosidad (ej. "soy magnífico"), b) esfuerzo por ser único (ej. "muestro a los demás lo especial que soy"), y c) encanto (ej. "consigo ser el centro de atención con mis magníficas aportaciones"). En el caso de rivalidad, esta está conformada por: a) devaluación (ej. "la mayoría de la gente no conseguirá nada"), b) esfuerzo por la supremacía (ej. "disfruto en secreto de los fracasos de mis rivales"), y c) agresividad (ej. "suele molestarme que me critiquen") (Back et al., 2013). La versión empleada fue la adaptación al español elaborada por Doroszuk et al. (2019), la cual presentó unos coeficientes de alfa de Cronbach adecuados, tanto para admiración (mínimo $\alpha=.78$; máximo $\alpha=.84$ ) como para rivalidad (mínimo $\alpha=.81$; máximo $\alpha=.85$ ). 


\section{Big Five Inventory (BFI)}

Es un cuestionario de 44 ítems de autorreporte, con una escala de respuesta tipo Likert, que va desde 1 (muy en desacuerdo) hasta 5 (muy de acuerdo). La primera versión en español fue presentada por Benet-Martínez y John (1998) y fue la utilizada para esta investigación. Los ítems se distribuyen a través de cinco escalas o factores denominados: extraversión (8 ítems; ej., "es extrovertido, sociable"), afabilidad ( 9 ítems; ej., "es considerado y amable con casi todos"), responsabilidad (9 ítems; ej., "es minucioso en el trabajo"), neuroticismo (8 ítems; ej., "se pone nervioso con facilidad") y apertura (10 ítems; ej. "es original, se le ocurren ideas nuevas"). En cuanto a la confiabilidad de los cinco factores, estos han presentado valores adecuados en extraversión $(\alpha=.79)$, responsabilidad $(\alpha=.70)$, neuroticismo $(\alpha=.74)$, y apertura $(\alpha=.76)$; sin embargo, la escala que mostró la confiabilidad más baja de todos fue afabilidad $(\alpha=.62)$ (Domínguez-Lara, Merino-Soto, Zamudio \& Guevara-Cordero, 2018).

\section{Escala de Autoestima de Rosenberg}

La escala consiste en 10 ítems que hacen referencia a la autoestima general. La versión en español fue desarrollada originalmente por Martín-Albo, Núñez, Navarro y Grijalvo (2007), con modificaciones realizadas posteriormente por Gómez-Lugo et al. (2016). Esta última fue la versión utilizada para esta investigación. Presenta una escala de respuesta tipo Likert de cuatro puntos, que van de 1 (totalmente en desacuerdo) hasta 4 (totalmente de acuerdo). La puntuación total de la escala se obtiene a través de la sumatoria simple de todas las respuestas, una vez que se han invertido las puntuaciones de los ítems 2 , 6, 8 y 9. Por lo que la puntuación mínima es de 10 y la máxima es de 40; puntuaciones más altas reflejan una autoestima general más elevada. Los coeficientes de confiabilidad alfa de Cronbach rondan desde .83 a .86 (Gómez-Lugo et al., 2016).

\section{Información sociodemográfica}

Las características sociodemográficas de los estudiantes fueron recabadas a partir de un cuestionario autoadministrado, donde se preguntaba la edad, el sexo y la carrera universitaria a la cual pertenecía el participante.

\section{Procedimiento}

La versión en línea de los cuestionarios fue realizada a partir de la plataforma Google Forms. Esta plataforma facilita la elaboración de cuestionarios en línea y de compartirlos a través de enlaces, que pueden enviarse por distintos medios (ej., redes sociales, correo electrónico y servicios de mensajería instantánea); asimismo permite registrar las respuestas de los participantes, las cuales pueden descargarse posteriormente como un archivo desde la misma plataforma.

En el cuestionario se incluyó un mensaje de bienvenida, similar al que se daría junto con las instrucciones en la versión de papel y lápiz, explicando los objetivos de la investigación, un estimado de la duración promedio para completar los cuestionarios y una clarificación acerca del carácter voluntario de su participación, así como del anonimato y la confidencialidad con la que se tratarían sus respuestas. Adicionalmente se especificó que los fines de la investigación eran únicamente científicos. 
El método que se empleó para poder acceder a la muestra fue el denominado bola de nieve, en el que se accede a algunos miembros que califican dentro de la muestra y a partir de ellos se contacta y accede a otros posibles participantes (Peña, 2017). En este caso, se contactó a estudiantes de distintas carreras de pregrado en la universidad para que completaran la encuesta, estos la compartieron con otros estudiantes y así sucesivamente hasta que finalizara el período de recolección, el cual se limitó a siete días desde el envío del primer cuestionario. Una vez culminado este plazo, se deshabilitó la opción de recibir nuevas entradas en la plataforma y se descargaron las respuestas finales.

\section{Resultados}

Con respecto a los coeficientes de confiabilidad para el NARQ, los resultados fueron similares a los encontrados en otros países hispanohablantes. En comparación con los resultados hallados en Chile, España y Colombia, presentados por Doroszuk et al. (2019), los índices alfa de Cronbach para las escalas generales fueron similares en la muestra venezolana. Específicamente, admiración narcisista presentó un valor alfa de .823 y rivalidad narcisista de .803. Estos valores reflejan una adecuada confiabilidad de las mediciones realizadas con ambas escalas, siendo además semejantes a los reportados por Doroszuk et al. (2019). Los índices de confiabilidad y los descriptivos de todos los instrumentos utilizados se presentan en la tabla 1.

Tabla 1.

Coeficientes de confiabilidad y estadísticos descriptivos para las estrategias generales y los subcomponentes del NARQ, los Cinco Factores de Personalidad (BFI) y Escala de Autoestima

\begin{tabular}{lccccc}
\hline Variable & $\boldsymbol{\alpha}$ & $\boldsymbol{M}$ & $\boldsymbol{D E}$ & $\mathbf{A s}$ & $\mathbf{K}$ \\
\hline 1. Admiración narcisista & .823 & 3.64 & .98 & -.03 & -.48 \\
$\quad$ Grandiosidad & .636 & 3.50 & 1.16 & -.27 & .03 \\
$\quad$ Esfuerzo por ser único & .673 & 3.90 & 1.20 & -.12 & -.73 \\
$\quad$ Encanto & .600 & 3.55 & 1.12 & -.27 & -.27 \\
2. Rivalidad narcisista & .803 & 2.07 & .80 & 1.27 & 2.14 \\
Devaluación & .755 & 1.56 & .87 & 2.17 & 5.46 \\
Esfuerzo por la supremacía & .722 & 1.94 & 1.10 & 1.32 & 1.52 \\
Agresividad & .638 & 2.71 & 1.04 & .69 & .49 \\
3. Apertura & .662 & 3.80 & .54 & -.22 & -.27 \\
4. Responsabilidad & .709 & 3.66 & .59 & -.07 & -.54 \\
5. Extraversión & .880 & 3.05 & .93 & .15 & -.58 \\
6. Afabilidad & .634 & 3.66 & .57 & -.40 & .17 \\
7. Neuroticismo & .797 & 3.30 & .80 & -.08 & -.76 \\
8. Autoestima & .859 & 27.70 & 5.78 & -.36 & -.01 \\
\hline
\end{tabular}

Notas: $\alpha=$ alfa de Cronbach; $M=$ media aritmética; $D E=$ desviación estándar; As = asimetría; $\mathrm{K}=$ curtosis. Todos los estadísticos fueron calculados $\operatorname{con} N=159$ para cada variable. 
Posteriormente, para realizar el análisis factorial exploratorio se empleó el programa Factor (Lorenzo-Seva \& Ferrando, 2013); específicamente, se empleó una matriz de correlaciones policórica al tratarse de variables ordinales, como sucede con las pruebas psicológicas con ítems tipo Likert (Lloret-Segura, Ferreres-Traver, Hernández-Baeza \& Tomás-Marco, 2014). Al incluir los 18 ítems del cuestionario, tanto la prueba KMO (0.858) como la prueba de esfericidad de $\operatorname{Bartlett}\left(\chi_{(153)}^{2}=1751.9 ; p<.001\right)$ indicaron la adecuación de los datos para realizar el análisis factorial. Mediante el método de análisis paralelo (Horn, 1965), se estimó el número de factores a extraer, hallándose solo dos factores cuyo porcentaje de varianza explicada superaba el percentil 95 de la varianza promedio explicada por azar, llegando a explicar estos dos factores el $54.8 \%$ de la varianza total.

Para la extracción de estos dos factores se empleó el método robusto de mínimos cuadrados no ponderados (RULS, por sus siglas en inglés), en conjunto con un método de rotación de factores Promin Robusto (Lorenzo-Seva \& Ferrando, 2018), siguiendo las recomendaciones de Lloret-Segura et al. (2014). La estructura factorial encontrada coincide con los resultados esperados (ver tabla 2), ya que los ítems 4, 6, 9, 10, 11, 12, 13, 14 y 17 cargaron en el primer factor, correspondiente a rivalidad narcisista; mientras que, por su parte, los ítems 1, 2, 3, 5, 7, 8, 15, 16 y 18 lo hicieron en el segundo factor, correspondiente a admiración narcisista. Cabe resaltar que dos de los ítems presentaron cargas factoriales altas con ambos factores: el ítem 4, correspondiente a rivalidad, y el ítem 18, correspondiente a admiración. El primero de ellos presentó cargas factoriales positivas y muy similares, pero ligeramente superior en su factor correspondiente; mientras que el segundo presentó cargas factoriales opuestas en cada factor, aunque mantuvo una carga positiva con su factor correspondiente. 
Tabla 2.

Matriz de cargas factoriales rotada para el NARQ

\begin{tabular}{lcc}
\hline Ítem & Factor 1 & Factor 2 \\
\hline 1. Soy magnífico & & .687 \\
2. Algún día seré famoso & .601 \\
3. Muestro a los demás lo especial que soy & .793 \\
4. Me molesta cuando otra persona me roba el protagonismo & .450 & .435 \\
5. Disfruto mucho de mis éxitos & & .657 \\
6. Disfruto en secreto de los fracasos de mis rivales & .715 & \\
7. Suelo ser capaz de atraer la atención de los demás hacia mí mismo & & .567 \\
$\quad$ durante una conversación & & .676 \\
8. Merezco que me consideren una gran persona & & \\
9. Quiero que mis rivales fracasen & .752 & \\
10. Disfruto cuando alguien es inferior a mí & .609 & .344 \\
11. Suele molestarme que me critiquen & .674 \\
12. Me cuesta soportar que otra persona sea la protagonista de un evento \\
13. La mayoría de la gente no conseguirá nada \\
14. Los demás no valen nada \\
15. Ser una persona muy especial me da mucha fuerza \\
16. Puedo ser el centro de atención gracias a mis excelentes aportaciones \\
17. La mayoría de la gente son perdedores de alguna forma \\
18. En general, se me da muy bien tratar con los demás \\
\hline
\end{tabular}

Nota: Se omitieron las cargas factoriales iguales o inferiores a 30 .

Con respecto a las correlaciones entre variables, se encontró que la estrategia de rivalidad se asocia de forma negativa con los rasgos de extraversión y afabilidad, así como con la autoestima. En el caso de la admiración, esta se asocia positivamente con extraversión, apertura a la experiencia, afabilidad y responsabilidad; finalmente, para la variable autoestima la asociación también resulta positiva. En la mayoría de los casos, estas correlaciones son bajas, exceptuando la asociación entre rivalidad y afabilidad $(r=-.45)$ la cual es moderada (ver tabla 3). 


\section{Tabla 3.}

Correlaciones producto-momento de Pearson para edad, NARQ, Escala de Autoestima y los Cinco Factores de Personalidad (BFI)

\begin{tabular}{lcccccccc}
\hline Variable & $\mathbf{2}$ & $\mathbf{3}$ & $\mathbf{4}$ & $\mathbf{5}$ & $\mathbf{6}$ & $\mathbf{7}$ & $\mathbf{8}$ & $\mathbf{9}$ \\
\hline 1. Edad & .010 & -.064 & .073 & .003 & .005 & $.191^{*}$ & -.060 & -.020 \\
2. Admiración & & $.175^{*}$ & $.425^{* *}$ & $.384^{* *}$ & $.165^{*}$ & $.160^{*}$ & -.045 & $.237^{* *}$ \\
3. Rivalidad & & & $-.249^{* *}$ & $-.285^{* *}$ & $-.450^{* *}$ & -.145 & .132 & -.114 \\
4. Autoestima & & & & $.544^{* *}$ & $.297^{* *}$ & $.462^{* *}$ & $-.422^{* *}$ & $.251^{* *}$ \\
5. Extraversión & & & & & $.363^{* *}$ & $.266^{* *}$ & $-.293^{* *}$ & $.333^{* *}$ \\
6. Afabilidad & & & & & & $.240^{* *}$ & $-.193^{*}$ & .143 \\
7. Responsabilidad & & & & & & $-.227^{* *}$ & $.169^{*}$ \\
8. Neuroticismo & & & & & & & & -.085 \\
9. Apertura & & & & & & & & - \\
\hline
\end{tabular}

Nota: Todas las correlaciones fueron calculadas con $N=159 . *$ La correlación es significativa en el nivel .05 (bilateral). **La correlación es significativa en el nivel .01 (bilateral).

En cuanto a las diferencias en función del sexo, no se hallaron variaciones significativas en las escalas generales de rivalidad y admiración; sin embargo, se halló significancia estadística al analizar las subescalas de esfuerzo por la supremacía, y devaluación, ambas pertenecientes a la escala general de rivalidad narcisista; la primera de ellas tomando un nivel de significancia de $5 \%$, y la segunda de $10 \%$ (ver tabla 4). Para las dos subescalas, los hombres presentaron una mayor puntuación media, aunque la magnitud de esta diferencia es baja. Es importante recalcar que la potencia estadística de estos contrastes es baja $(1-\beta<0.8)$, debido a las discrepancias entre la cantidad de personas de sexo masculino y femenino, por lo que deben ser interpretados con precaución y expandidos posteriormente en muestras balanceadas. 
Tabla 4.

Estadísticos descriptivos para las estrategias generales y los subcomponentes del NARQ en español, y prueba de diferencia de medias por sexo

\begin{tabular}{|c|c|c|c|c|c|c|c|}
\hline Variable & Sexo $^{a}$ & $M[$ IC95 \%] & $D E$ & $t(g l)$ & Sig. & $d$ & $1-\beta$ \\
\hline \multirow{2}{*}{$\begin{array}{l}\text { 1. Admiración } \\
\text { narcisista }\end{array}$} & Masculino & $3.79[3.47-4.15]$ & 1.06 & \multirow{2}{*}{$1.110(157)$} & \multirow{2}{*}{.269} & \multirow{2}{*}{0.201} & \multirow{2}{*}{0.196} \\
\hline & Femenino & $3.59[3.42-3.76]$ & .94 & & & & \\
\hline \multirow{2}{*}{ Grandiosidad } & Masculino & $3.66[3.31-4.02]$ & 1.16 & \multirow{2}{*}{$1.093(157)$} & \multirow{2}{*}{.276} & \multirow{2}{*}{0.198} & \multirow{2}{*}{0.192} \\
\hline & Femenino & $3.43[3.22-3.64]$ & 1.15 & & & & \\
\hline \multirow{2}{*}{$\begin{array}{l}\text { Esfuerzo por ser } \\
\text { único }\end{array}$} & Masculino & $4.04[3.65-4.43]$ & 1.27 & \multirow{2}{*}{$.948(157)$} & \multirow{2}{*}{.345} & \multirow{2}{*}{0.167} & \multirow{2}{*}{0.15} \\
\hline & Femenino & $3.84[3.63-4.05]$ & 1.18 & & & & \\
\hline \multirow{2}{*}{ Encanto } & Masculino & $3.66[3.30-4.03]$ & 1.20 & \multirow{2}{*}{.765 (157) } & \multirow{2}{*}{.446} & \multirow{2}{*}{0.139} & \multirow{2}{*}{0.118} \\
\hline & Femenino & $3.51[3.31-3.71]$ & 1.09 & & & & \\
\hline \multirow{2}{*}{$\begin{array}{l}\text { 2. Rivalidad } \\
\text { narcisista }\end{array}$} & Masculino & $2.17[1.89-2.45]$ & .92 & \multirow{2}{*}{.899 (157) } & \multirow{2}{*}{.370} & \multirow{2}{*}{0.163} & \multirow{2}{*}{0.145} \\
\hline & Femenino & 2.04 [1.91-2.18] & .75 & & & & \\
\hline \multirow{2}{*}{ Devaluación } & Masculino & 1.79 [1.43-2.15] & 1.17 & \multirow{2}{*}{$1.865^{b}(157)$} & \multirow{2}{*}{.064} & \multirow{2}{*}{0.338} & \multirow{2}{*}{0.457} \\
\hline & Femenino & 1.49 [1.36-1.62] & .73 & & & & \\
\hline \multirow{2}{*}{$\begin{array}{l}\text { Esfuerzo por la } \\
\text { supremacía }\end{array}$} & Masculino & 2.24 [1.89-2.59] & 1.14 & \multirow{2}{*}{2.005 (157) } & \multirow{2}{*}{.047} & \multirow{2}{*}{0.363} & \multirow{2}{*}{0.51} \\
\hline & Femenino & $1.84[1.65-2.03]$ & 1.07 & & & & \\
\hline \multirow{2}{*}{ Agresividad } & Masculino & $2.49[2.16-2.81]$ & 1.07 & \multirow{2}{*}{$-1.607(157)$} & .110 & 0.291 & 0.358 \\
\hline & Femenino & $2,79[2,60-2,97]$ & 1,02 & & & & \\
\hline
\end{tabular}

Notas: $M$ [IC95 \%] = media aritmética con intervalo de confianza al $95 \% ; D E=$ desviación estándar; $t=$ prueba $\mathrm{t}$ de Student para grupos independientes; $\mathrm{gl}=$ grados de libertad; Sig. = significancia estadística bilateral; $d=$ coeficiente de magnitud de efecto $d$ de Cohen; $1-\beta=$ potencia estadística de la prueba. ${ }^{\mathrm{a}}$ Femenino $n=118$, Masculino $n=41$. ${ }^{\mathrm{b}}$ No se asumen varianzas iguales a partir de la prueba de Levene.

Al comparar los resultados obtenidos en la muestra venezolana con los datos provenientes de España, Chile y Colombia (Doroszuk et al., 2019), se observaron las siguientes variaciones: en cuanto a admiración narcisista, se hallaron diferencias significativas entre los países de procedencia, a pesar de que la magnitud de esta fue baja ( $\eta^{2}$ =0.06). Específicamente, la muestra venezolana presentó mayores niveles de admiración que la española, pero inferiores a los presentados en Colombia. En comparación con los datos de Chile, no hubo diferencias significativas con los obtenidos en Venezuela (ver tabla 5).

Para la rivalidad narcisista, nuevamente se hallaron diferencias significativas, a pesar de que la magnitud de esta sea baja $\left(\eta^{2}=0.016\right)$. Sin embargo, el patrón en esta ocasión fue diferente: los datos de la muestra venezolana solo presentaron diferencias con la muestra colombiana, ya que estos últimos cuentan con valores más bajos de rivalidad. Con la muestra chilena y la muestra española, los datos obtenidos en Venezuela no se diferenciaron significativamente. 
Tabla 5.

Estadísticos descriptivos y prueba ANOVA para rivalidad y admiración narcisista entre muestras españolas, chilenas, colombianas y venezolanas

\begin{tabular}{|c|c|c|c|c|c|c|c|}
\hline Variable & País $^{a}$ & $M[$ IC95 \%] & $D E$ & $N$ & $F(g l)$ & Sig. & $\eta^{2}$ \\
\hline \multirow{4}{*}{$\begin{array}{l}\text { Admiración } \\
\text { narcisista }\end{array}$} & España & $3.44[3.36-3.52]$ & .73 & 310 & & \multirow{4}{*}{$<.001$} & \multirow{4}{*}{.06} \\
\hline & Chile & $3.60[3.49-3.71]$ & .85 & 234 & 21.55 & & \\
\hline & Colombia & $3.99[3.89-4.09]$ & .79 & 256 & (3.955) & & \\
\hline & Venezuela & $3.64[3.49-3.80]$ & .98 & 159 & & & \\
\hline \multirow{4}{*}{$\begin{array}{l}\text { Rivalidad } \\
\text { narcisista }\end{array}$} & España & $2.10[2.02-2.19]$ & .76 & 310 & & \multirow{4}{*}{$<.001$} & \multirow{4}{*}{.016} \\
\hline & Chile & $2.02[1.92-2.12]$ & .79 & 234 & 5.27 & & \\
\hline & Colombia & $1.86[1.78-1.94]$ & .65 & 256 & (3.955) & & \\
\hline & Venezuela & $2.07[1.95-2.19]$ & .80 & 159 & & & \\
\hline
\end{tabular}

Notas: $M$ [IC95\%] = media aritmética con intervalo de confianza al $95 \% ; D E=$ desviación estándar; $N=$ tamaño muestral; $F=$ prueba $F$ de Fisher para grupos independientes; $g l=$ grados de libertad (numerador/denominador); Sig. = significancia estadística bilateral; $\eta^{2}=$ coeficiente de magnitud de efecto eta-cuadrado. ${ }^{a}$ Los datos pertenecientes a España, Chile y Colombia fueron tomados de Doroszuk et al. (2019).

\section{Discusión}

El objetivo principal de la investigación fue realizar una primera aproximación para comprobar la adecuación psicométrica del Cuestionario de Admiración y Rivalidad Narcisista (NARQ) en su versión en español desarrollada por Doroszuk et al. (2019), aplicada en una muestra de estudiantes universitarios venezolanos.

Con respecto a los índices de confiabilidad del cuestionario, se pudo apreciar que tanto la escala de rivalidad como la de admiración se consideran medidas confiables, tomando como criterio un valor mínimo de .80 para el coeficiente alfa de Cronbach; sin embargo, la mayoría de las subescalas del cuestionario no alcanzaron niveles mínimos de confiabilidad de .70. Estas diferencias pueden deberse a la reducida cantidad de ítems que componen cada subescala, que, al ser sumadas, permiten un incremento de la confiabilidad de las respectivas escalas generales (DeVon et al., 2007): siendo que cada una de las subescalas cuenta con tres ítems, para un total de nueve ítems por escala. Por tanto, se pueden considerar como confiables los resultados para las escalas de rivalidad y admiración; mientras que las subescalas deben ser analizadas con precaución.

Los análisis factoriales llevados a cabo con la escala de admiración y rivalidad se ajustaron a los resultados reportados Back et al. (2013), si bien las técnicas específicas del análisis factorial exploratorio fueron diferentes, para ajustarse a las prácticas recomendadas para este tipo de variables (Lloret-Segura et al., 2014). A pesar de estas diferencias, todos los ítems analizados cargaron en sus respectivos factores de rivalidad y admiración narcisista.

Al profundizar en la asociación entre las puntuaciones obtenidas y las variables que, de acuerdo con la literatura, han presentado patrones de correlación particular, los resultados pueden considerarse adecuados. Entre estos, la autoestima ha sido una variable usualmente asociada con el narcisismo (Geukes et al., 2017); a pesar de esto, existen múltiples resultados contradictorios en la dirección de la relación entre ellas. Como puede observarse en los 
resultados obtenidos, dos estrategias consideradas como narcisistas presentan relaciones diametralmente opuestas con la autoestima: una relación positiva con la admiración y una relación negativa con la rivalidad, tal como reportaron Doroszuk et al. (2019).

La principal diferencia que se pudo evidenciar en cuanto a la relación de los cinco factores de personalidad con las estrategias narcisistas, de acuerdo a los resultados reportados originalmente por Back et al. (2013), estriba en el papel de la afabilidad y la admiración narcisista. En principio, estos autores encontraron que la relación entre ambas variables no era significativa, sin embargo, en esta oportunidad se pudo evidenciar la presencia de una relación positiva y significativa entre ambas. Además de esta discrepancia, el patrón de asociaciones fue similar al esperado, donde la rivalidad narcisista se asocia con la introversión y el antagonismo, mientras que la admiración lo hace con los rasgos de extroversión y la apertura a la experiencia, resultados semejantes a los obtenidos por Leckelt et al. (2017).

Otra de las principales diferencias halladas se refiere al papel particular del sexo en cuanto a la rivalidad y a la admiración; pero debe tomarse en cuenta que incluso en la literatura estas discrepancias parecen estar presentes. En su investigación intercultural, Doroszuk et al. (2019) analizan las diferencias entre hombres y mujeres tanto en la rivalidad como en la admiración narcisista y hallaron que en Chile, Colombia y España los hombres presentan un mayor nivel de rivalidad, en comparación con las mujeres. Solo en España se halló este mismo patrón a favor de los hombres en cuanto a la admiración, ya que en los otros dos países no se apreciaron diferencias.

Existen diferencias culturales en cuanto a la predominancia entre hombres y mujeres de las estrategias narcisistas, ya que usualmente los hombres muestran una mayor prevalencia del uso de la rivalidad (Doroszuk et al., 2019). En el caso de la muestra venezolana, no se pudo apreciar este patrón particular en las estrategias generales; sin embargo, ahondando en los componentes de la rivalidad, se repitió el estándar mostrado en otros países, donde los hombres presentan mayores elementos de devaluación y de esforzarse por la supremacía (ambos elementos de rivalidad narcisista), al ser comparados con las mujeres. Debe tomarse en consideración que, al analizar la potencia estadística de las comparaciones por sexo, esta se encuentra por debajo de los niveles ideales, por lo que se pueden estar obteniendo resultados falsamente negativos, debidos al reducido tamaño muestral (Peña, 2017). Por tanto, el patrón venezolano parece ser similar al encontrado en otros países, una vez que se analizan los componentes de la rivalidad narcisista, aunque es necesario ampliar el tamaño de la muestra y balancear los grupos a comparar para contar con la potencia estadística suficiente, para así identificar estas posibles diferencias por sexo. 


\section{Conclusión}

El Cuestionario de Admiración y Rivalidad Narcisista es un instrumento de medición que se encuentra cada vez más presente en la investigación de la personalidad narcisista. Al tratarse de un cuestionario corto permite obtener información relevante en poco tiempo y con relativa facilidad. Su utilidad radica en que facilita medir simultáneamente dos estrategias narcisistas documentadas en la investigación, las cuales influyen en la manera de relacionarse con las demás personas y están asociadas usualmente con consecuencias sociales particulares, a corto y largo plazo.

Como muestra de su relevancia actual se encuentran las adaptaciones del cuestionario en diferentes idiomas. Esta línea de investigación ha llegado incluso a las regiones hispanohablantes, las cuales ya tienen acceso a una adaptación en español. A pesar de esto, algunos países latinoamericanos aún no cuentan con un estudio particular para asegurar la adecuación del instrumento para su población. En esta investigación se propuso aportarle a la población venezolana un estudio preliminar que permitiera dar indicios de que el NARQ cumple con los estándares de confiabilidad y validez para ser empleado en contextos de investigación y de práctica aplicada. Este objetivo se considera alcanzado, siendo este entonces el punto de partida para la inclusión del NARQ como una herramienta disponible para los investigadores y profesionales interesados en el estudio de la personalidad narcisista, así como para futuras investigaciones que profundicen en sus propiedades psicométricas en la población venezolana.

\section{Referencias}

American Psychiatric Association. (1980). Diagnostic and statistical manual of mental disorders (3ra ed.). Washington: American Psychiatric Association.

Back, M. D. (2018). The narcissistic admiration and rivalry concept. En A. D. Hermann, A. B. Brunell \& J. D. Foster (Eds.), Handbook of trait narcissism: Key advances, research methods, and controversies (pp. 57-67). Cham: Springer. doi: https://doi.org/10.1007/978-3-319-92171-6_6

Back, M. D., Küfner, A. C., Dufner, M., Gerlach, T. M., Rauthmann, J. F., \& Denissen, J. J. (2013). Narcissistic admiration and rivalry: Disentangling the bright and dark sides of narcissism. Journal of Personality and Social Psychology, 105(6), 1013-1037. doi: http://dx.doi.org/10.1037/a0034431

Benet-Martínez, V. \& John, O. P. (1998). Los Cinco Grandes across cultures and ethnic groups: Multitrait-multimethod analyses of the Big Five in Spanish and English. Journal of Personality and Social Psychology, 75(3), 729-750. doi: https://doi.org/10.1037/0022-3514.75.3.729

Benson, A. J., Jeschke, J., Jordan, C. H., Bruner, M. W., \& Arnocky, S. (2018). Will they stay or will they go? Narcissistic admiration and rivalry predict ingroup affiliation and devaluation. Journal of Personality, 87(4), 871-888. doi: https://doi.org/10.1111/jopy.12441

DeVon, H. A., Block, M. E., Moyle-Wright, P., Ernst, D. M., Hayden, S. J., Lazzara, D. J., ... \& Kostas-Polston, E. (2007). A psychometric toolbox for testing validity and reliability. Journal of Nursing Scholarship, 39(2), 155-164. 
Domínguez-Lara, S., Merino-Soto, C., Zamudio, B., \& Guevara-Cordero, C. (2018). Big Five Inventory en universitarios peruanos: Resultados preliminares de su validación. Psykhe, 27(2), 1-12. doi: https://doi.org/10.7764/psykhe.27.2.1052

Doroszuk, M., Kwiatkowska, M. M., Torres-Marín, J., Navarro-Carrillo, G., Włodarczyk, A., Blasco-Belled, A., ... \& Rogoza, R. (2019). Construct validation of the Narcissistic Admiration and Rivalry Questionnaire in Spanish-speaking countries: Assessment of the reliability, structural and external validity and cross-cultural equivalence. International Journal of Psychology, 55(3), 413-424. doi: https://doi.org/10.1002/ijop.12595

Emmons, R. A. (1987). Narcissism: Theory and measurement. Journal of Personality and Social Psychology, 52(1), 11-17. doi: https://doi.org/10.1037/0022-3514.52.1.11

Geukes, K., Nestler, R. H., Dufner, M., Küfner, A. C. P, Egloff, B., Denissen, J. J. A., \& Back, M. D. (2017). Puffed-up but shaky selves: State self-esteem level and variability in narcissists. Journal of Personality and Social Psychology, 112(5), 769786. doi: https://doi.org/10.1037/pspp0000093

Gómez-Lugo, M., Espada, J. P., Morales, A., Marchal-Bertrand, L., Soler, F., \& VallejoMedina, P. (2016). Adaptation, validation, reliability and factorial equivalence of the Rosenberg self-esteem scale in Colombian and Spanish population. The Spanish Journal of Psychology, 19: e66. doi: http://dx.doi.org/10.1017/sjp.2016.67

Hair, J. F., Black, W. C., Babin, B. J., \& Anderson, R. E. (2009). Multivariate data análisis (7ma ed.). Pearson Education.

Hendin, H. M. \& Cheek, J. M. (1997). Assessing hypersensitive narcissism: A reexamination of Murray's Narcism Scale. Journal of Research in Personality, 31(4), 588-599. doi: https://doi.org/10.1006/jrpe.1997.2204

Horn, J. L. (1965). A rationale and test for the number of factors in a factor analysis. Psychometrika, 30, 179-185.

Leckelt, M., Wetzel, E., Gerlach, T. M., Ackerman, R. A., Miller, J. D., Chopik, W. J., Penke, L., Geukes, K., Küfner, A. C. P., Hutteman, R., Richter, D., Renner, K.-H., Allroggen, M., Brecheen, C., Campbell, W. K., Grossmann, I., \& Back, M. D. (2017). Validation of the Narcissistic Admiration and Rivalry Questionnaire Short Scale (NARQ-S) in convenience and representative samples. Psychological Assessment, 30(1), 86-96. doi: https://doi.org/10.1037/pas0000433

Levy K. N., Ellison, W. D., \& Reynoso, J. S. (2011). A historical review of narcissism and narcissistic personality. En W. K. Campbell \& J. D. Miller (Eds.). The handbook of narcissism and narcissistic personality disorder (pp. 1-13). New Jersey: John Wiley \& Sons.

Lloret-Segura, S., Ferreres-Traver, A., Hernández-Baeza, A., \& Tomás-Marco, I. (2014). El análisis factorial exploratorio de los ítems: una guía práctica, revisada y actualizada. Anales de Psicología, 30(3), 1151-1169. doi: http://dx.doi.org/10.6018/analesps.30.3.199361

Lorenzo-Serva, U. \& Ferrando, P. J. (2018). Robust Promin: un método para la rotación de factores de diagonal ponderada. Liberabit, Revista Peruana de Psicología, 25(1), 99106. doi: https://doi.org/10.24265/liberabit.2019.v25n1.08 
Lorenzo-Seva, U., \& Ferrando, P. J. (2013). FACTOR 9.2: A comprehensive program for fitting exploratory and semiconfirmatory factor analysis and IRT models. Applied Psychological Measurement, 37(6), 497-498. doi: https://doi.org/10.1177/0146621613487794

Martín-Albo, J., Núñez, J. L., Navarro, J. G., \& Grijalvo, F. (2007). The Rosenberg SelfEsteem Scale: Translation and validation in university students. The Spanish Journal of Psychology, 10(2), 458-467.

Peña, G. (2017). Estadística inferencial: Una introducción para las ciencias del comportamiento (2da ed.). Caracas: AB ediciones.

Pincus, A. L. \& Roche, M. J. (2011). Narcissistic grandiosity and narcissistic vulnerability. En W. K. Campbell y J. D. Miller (Eds.). The handbook of narcissism and narcissistic personality disorder (pp. 31-40). New Jersey: John Wiley \& Sons.

Raskin, R. \& Terry, H. (1988). A principal components analysis of the Narcissistic Personality Inventory and further evidence of its construct validity. Journal of Personality and Social Psychology, 54(5), 890-902. doi: https://doi.org/10.1037/0022-3514.54.5.890

Raskin, R. N. \& Hall, C. S. (1979). A narcissistic personality inventory. Psychological Reports, 45(2), 590-590. doi: https://doi.org/10.2466/pr0.1979.45.2.590

Rogoza, R., Rogoza, M., \& Wyszynska, P. (2016). Polish adaptation of the narcissistic admiration and rivalry concept. Pol. Forum Psychol. 21, 410-431. doi: https://doi.org/10.14656/PFP20160306

Rogoza, R., Zemojtel-Piotrowska, M., \& Campbell, W. K. (2019). Measure of narcissism: From classical applications to modern approaches. Studia Psychologica: Teoria et Praxis, 18(1), 27-48. doi: https://doi.org/10.21697/sp.2018.18.1.02

Tyler, I. (2007). From "the me decade" to "the me millennium": The cultural history of narcissism. International Journal of Cultural Studies, 10(3), 343-363. doi: https://doi.org/10.1177/1367877907080148

Vecchione, M., Dentale, F., Graziano, M., Dufner, M., Wetzel, E., Leckelt, M., \& Back, M. D. (2018). An Italian validation of the Narcissistic Admiration and Rivalry Questionnaire (NARQ): Further evidence for a two-dimensional model of grandiose narcissism. BPA-Applied Psychology Bulletin, 66(281), 29-37.

Wink, P. (1991). Two faces of narcissism. Journal of Personality and Social Psychology, 61(4), 590-597. doi: https://doi.org/10.1037/0022-3514.61.4.590

Wurst, S. N., Gerlach, T. M., Dufner, M., Rauthmann, J. F., Grosz, M. P., Küfner, A. C. P., Denissen, J. J. A., \& Back, M. D. (2017). Narcissism and romantic relationships: The differential impact of narcissistic admiration and rivalry. Journal of Personality and Social Psychology, 112(2), 280-306. doi: http://dx.doi.org/10.1037/pspp0000113

Contribución de los autores: a) Concepción y diseño del trabajo; b) Adquisición de datos; c) Análisis e interpretación de datos; d) Redacción del manuscrito; e) revisión crítica del manuscrito.

D. C. J. ha contribuido en a, b, c, d, e.

Editora científica responsable: Dra. Cecilia Cracco. 